\title{
Effects of intensity and frequency on the perception of the time interval of tone burst sequence
}

\author{
Kenji Kurakata \\ National Institute of Bioscience and Human-Technology, 1-1, Higashi, Tsukuba, 305 Japan \\ (Received 15 June 1996)
}

Keywords : Time perception, Effects of intensity and frequency, Perception of music, Computer music, Auditory display PACS number: 43. 66. Lj, 43. 66. $\mathrm{Mk}$

\section{Introduction}

Time perception is an important aspect of our auditory function. Although many studies have focused on the perception of empty intervals delimited by markers (for review, see Fraisse, 1978, 1984), there seem to remain two problems to be investigated.

First, it has often been assumed that the markers (e.g., clicks or tone bursts) merely serve to indicate time intervals and do not affect time perception itself. This assumption may be reasonable, if the purpose of the study is to investigate the basic mechanism of time perception. The sounds we encounter in daily life, however, consist of various kinds of components differing in intensity and frequency. Therefore, when we consider the perception of these sounds, attention must be paid to the effect of characteristics of the markers.

Second, even when characteristics of the markers have been taken into account, their effects on time perception has still not been made clear. For example, while Triplett (1931) suggests that an interval defined with high-frequency tones is perceived to be shorter than one defined with low-frequency tones, there seems to be no study which shows any systematic effects of intensity. A possible reason for this is related to the configuration of the stimulus: the effect of intensity observed in single interval may be small. However, if the intervals were repeated successively, the effect would accumulate and become great enough to be detected. Moreover, a stimulus configuration of repeated intervals is more appropriate when we consider, for example, the perception of music.

To investigate the effects of the intensity and frequency of markers on the perceived time interval, the following experiments were conducted. In these experiments, sequences of tone bursts were used, instead of the single time intervals which were common in previous studies of time perception.

\section{Experiment 1 : Effect of intensity}

\subsection{Method}

One stimulus sequence consisted of equally spaced eight tone bursts (Fig. 1). The duration of each burst was $50 \mathrm{~ms}$ with $5 \mathrm{~ms}$ rise and fall time, and its frequency was $125,250,1,000$ or $4,000 \mathrm{~Hz}$. The onset-to-onset interval between the tone bursts of the standard stimulus (IS) was 300 or $600 \mathrm{~ms}$. The sound pressure level of the standard stimulus was $30,40,60$ or $80 \mathrm{~dB}$, and that of the comparison stimulus was always $60 \mathrm{~dB}$. (Three standard stimulus conditions, a $125-\mathrm{Hz}$ tone of 30 and $40 \mathrm{~dB}$ and a $250-\mathrm{Hz}$ tone of $30 \mathrm{~dB}$, were not used, since they were nearly inaudible.)

These stimuli were diotically presented to subjects in a soundproof room via headphones (STAX, SR- $\Lambda$ pro). The presented levels of the stimuli were adjusted using a measuring amplifier (B\&K, type 2610) and an artificial ear (B\&K, type 4153) with an adapter (B\&K, DB 0843).

The standard stimulus was presented first and followed by the comparison stimulus, as shown in Fig. 1. Subjects listened to the whole sequence first, and then adjusted the onset-to-onset intervals of the comparison stimulus (ICs) to the ISs so that both intervals were perceptually equal. ICs were changed proportionally (i.e., the intervals were kept equal to one another) as the subjects operated control buttons. Subjects could listen to the sequence as many times as they liked. Two trials were performed for every stimulus condition after training.

Three subjects with normal hearing ability participated in the experiment.

2.2 Results

The values of the two adjustments were averaged to calculate the PSE of each subject. The mean underestimations and standard deviations of the three subjects are shown in Fig. 2. The amount of underestimation was calculated by subtracting the mean PSE of the standard stimulus of $60 \mathrm{~dB}$ from that of each level.

The figure shows that the interval of the standard stimulus was underestimated as the level of tone bursts decreased. When the IS was $300 \mathrm{~ms}$ with $1,000-\mathrm{Hz}$ tones, the difference in underestimation between 30 and $80 \mathrm{~dB}$ amounted to $3.4 \%$ of the IS.

There seems to be no consistent interaction with the frequency of tone bursts. 


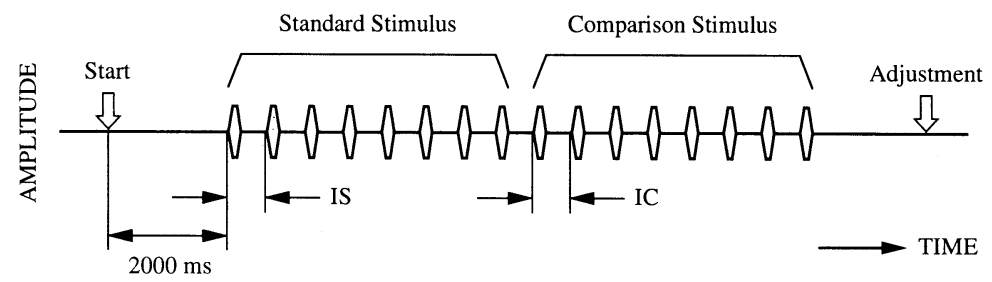

Fig. 1 Schematic illustration of the stimulus configuration used in the experiments. IS, interval of standard stimulus ; IC, interval of comparison stimulus.

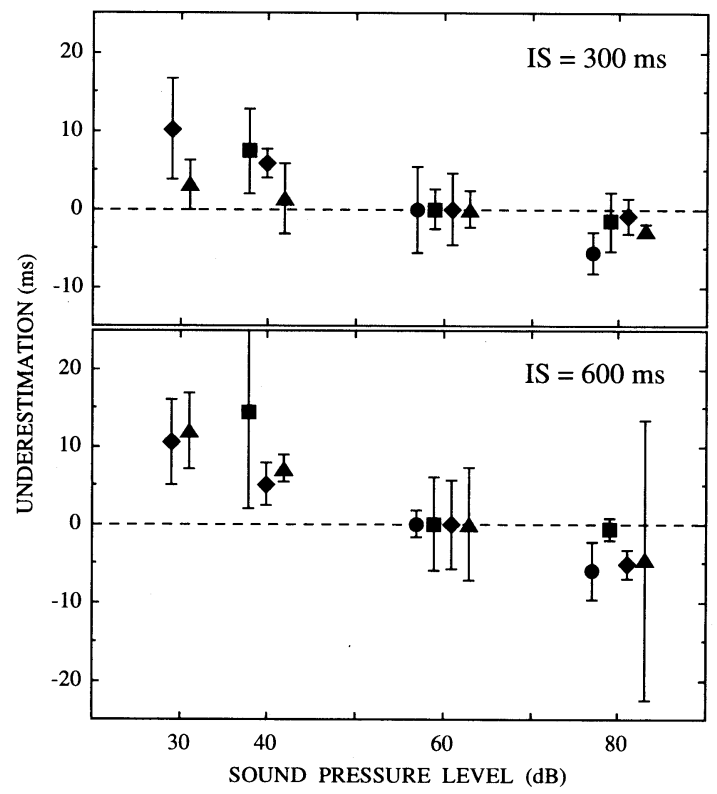

Fig. 2 Mean underestimations and standard deviations of the interval of tone burst sequence as a function of sound pressure level. $125 \mathrm{~Hz} ; \mathbf{\square}, 250 \mathrm{~Hz} ; \diamond, 1,000 \mathrm{~Hz} ; \boldsymbol{\Delta}, 4,000 \mathrm{~Hz}$.

\section{Experiment 2 : Effect of frequency}

\subsection{Method}

The stimulus configuration and experimental procedure were identical to those in Experiment 1, except that the frequency of tones was varied as an independent variable. The sound pressure level of the tone burst was $30,40,60$ or $80 \mathrm{~dB}$. IS was 300 or $600 \mathrm{~ms}$. The frequency of the standard stimulus was 125, 250, 1,000 or $4,000 \mathrm{~Hz}$, and that of the comparison stimulus was always $1,000 \mathrm{~Hz}$. (Three standard stimulus conditions, a $125-\mathrm{Hz}$ tone of 30 and $40 \mathrm{~dB}$ and a $250-\mathrm{Hz}$ tone of $30 \mathrm{~dB}$, were not used, since they were nearly inaudible.)

Subjects were required to adjust the ICs to the ISs so that both intervals were perceptually equal. The subjects were the same three who participated in Experiment 1. They performed two trials for every stimulus condition after training.

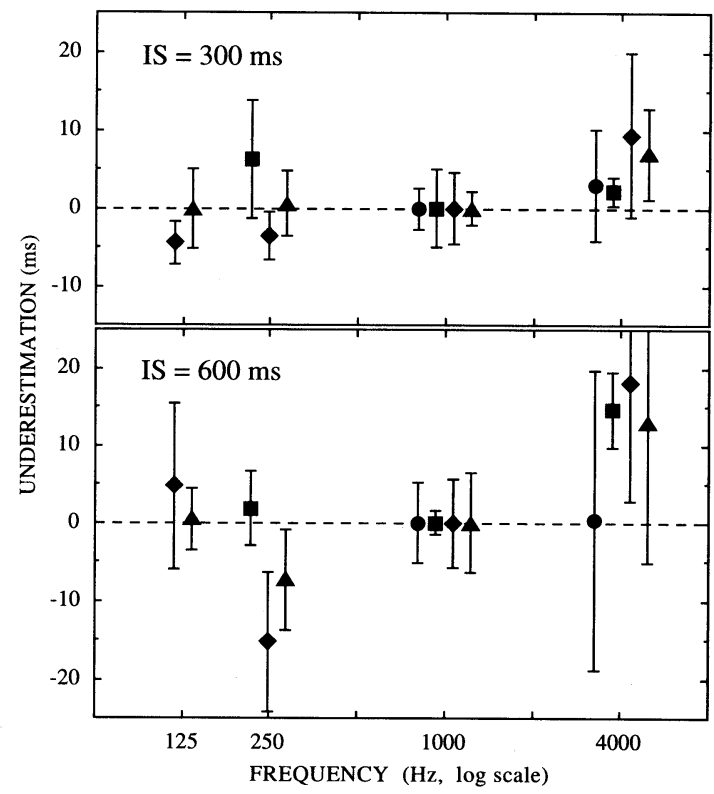

Fig. 3 Mean underestimations and standard deviations of the interval of tone burst sequence as a function of frequency. $\boldsymbol{Q}, 30$ $\mathrm{dB} ; \mathbf{\square}, 40 \mathrm{~dB} ; \bullet, 60 \mathrm{~dB} ; \boldsymbol{\Delta}, 80 \mathrm{~dB}$.

\subsection{Results}

The values of the two adjustments were averaged to calculate the PSE of each subject. The mean underestimations and standard deviations of the three subjects are shown in Fig. 3. The amount of underestimation was calculated by subtracting the mean PSE of the standard stimulus of $1,000 \mathrm{~Hz}$ from that of each frequency.

The figure shows that the sequences of $4,000-\mathrm{Hz}$ tones were underestimated. The amount of underestimation reached about $3 \%$ at its maximum for both durations of the IS. In the case of $125-$ and $250-\mathrm{Hz}$ tones, the underestimation was small or negative (i.e., the time interval was overestimated). Thus, the rate of the sequence of $4,000-\mathrm{Hz}$ tones was perceived to be faster than that of the low-frequency tones.

There seems to be no consistent interaction with the intensity of tone bursts. 


\section{K. KURAKATA : PERCEPTION OF TONE BURST SEQUENCE}

\section{General discussion}

The results of both experiments suggest that the rate of a tone burst sequence of low intensity or high frequency tends to be perceived as faster than it actually is. If these results reflect general characteristics of our auditory perception, they may also relate to the perception of music. For example, it is often said that computer music sounds awkward and unnatural. This phenomenon may be partly explained by the discrepancy between notated time intervals and perceived intervals which is caused by difference in the intensity or frequency of tones.

Furthermore, these two factors, intensity and frequency of tones, are not psychoacoustically independent of each other. As shown in the equal-loudness contours (ISO 226), our auditory system is most sensitive to tones with a frequency of around $4,000 \mathrm{~Hz}$. Even if a sound has a broadband spectrum, when its overall level is low, only the components around this frequency region are perceptually dominant. Therefore, if the effects of intensity and frequency are additive, a lower level of sound would combine with the perceptual dominance of high frequency components to accelerate the perceived rate of the sequence. This would result in more significant underestimation of intervals than Figs. 2 and 3 indicate. This phenomenon may be observed in classical music with large dynamics as played by an orchestra, or in popular music fading out at the ending.

Finally, these findings with respect to perceptual rate give some caution to the application of auditory display (AD ; e.g., Kramer, 1994). AD is a human-machine interface which operates by means of nonverbal sounds, instead of or in aid of visual display. For communication with high precision, it is desirable that the sounds used be carefully tuned to rectify the difference between physical and perceptual rates of presentation. The results of the present study would provide a basis for designing ADs.

Acknowledgement

This study was supported in part by a Grant-in-Aid for JSPS Fellows.

\section{References}

Fraisse, P. (1978). "Time and rhythm perception," in Handbook of Perception VIII, Perceptual Coding, E. C. Carterette and M. P. Friedman, Eds. (Academic Press, New York), Chap. 6, pp. 203-254.

Fraisse, P. (1984). "Perception and estimation of time," Ann. Rev. Psychol. 35, 1-36.

Kramer, G. (1994). "An Introduction to Auditory Display," in Auditory Display: Sonification, Audification, and Auditory Interfaces, G. Kramer, Ed. (Addison Wesley, Reading, MA), pp. 1-77.

Triplett, D. (1931). "The relation between the physical pattern and the reproduction of short temporal intervals : A study in the perception of filled and unfilled time," Psychol. Monogr. 41, 201-265. 